\title{
Downregulation of Notch1 inhibits the invasion of human hepatocellular carcinoma HepG2 and MHCC97H cells through the regulation of PTEN and FAK
}

\author{
YAN-JIAN HU ${ }^{1}$, HONG-YING LI ${ }^{2}$, KAI-JIE QIU ${ }^{3}$, DA-CHUAN LI ${ }^{3}$, \\ JIA-HUI ZHOU ${ }^{4}$, YAN-HUA HU ${ }^{3}$ and FENG-MIN ZHANG ${ }^{5}$
}

${ }^{1}$ Department of Gastroenterology, Second Affiliated Hospital of Harbin Medical University, Harbin, Heilongjiang 150086;
${ }^{2}$ Department of Biochemistry, Heilongjiang University of Chinese Medicine, Harbin, Heilongjiang 150040;
${ }^{3}$ Department of General Surgery, Second Affiliated Hospital of Harbin Medical University, Harbin,
Heilongjiang 150086; ${ }^{4}$ Department of Applied Statistics, Liaoning University, Shenyang, Liaoning 110036;
${ }^{5}$ Department of Microbiology, Harbin Medical University, Harbin, Heilongjiang 150086, P.R. China

Received April 7, 2014; Accepted August 8, 2014

DOI: 10.3892/ijmm.2014.1889

\begin{abstract}
Tumor invasion and metastasis are the main causes of mortality in patients with hepatocellular carcinoma (HCC). Thus, the effective inhibition of these tumorigenic processes is critical in order for HCC therapy to be effective. Previous studies have demonstrated that Notch1 is associated with metastasis in several human malignancies. However, the exact molecular mechanisms underlying the Notch1-mediated induction of the invasion of HCC cells remain poorly understood. In the present study, we demonstrate that, compared to the normal liver cell line, L02, Notch1 is highly expressed in the human HCC cell lines, HepG2 and MHCC97H. Using small interfering RNA (siRNA), we knocked down the expression of Notch1 in the cell lines. Notch1 expression in the HCC cell lines was also measured following transfection with siRNA using RT-PCR and western blot analysis. In addition, a migration and invasion assay was performed to determine the effects of Notch1 knockdown on cell migration and invasion. Our results demonstrated that the downregulation of Notch1 by small interfering RNA (siRNA) significantly inhibited the migration and invasion of both HCC cell lines. Additionally, we demonstrated that the knockdown of Notch1 in both HCC cell lines increased both the total expression of phosphatase and tensin homolog (PTEN) and its phosphorylated form. By contrast, focal adhesion kinase (FAK) and phospho-FAK expression was decreased following Notch1 depletion. Taken
\end{abstract}

Correspondence to: Dr Yan-Hua Hu, Department of General Surgery, Second Affiliated Hospital of Harbin Medical University, Harbin, Heilongjiang 150086, P.R. China

E-mail: drhuyanhua@163.com

Key words: Notch1, small interfering RNA, invasion, mechanism, hepatocellular carcinoma together, our data suggest that targeting Notch1 may be a useful therapeutic approach to inhibiting the metastasis of HCC cells.

\section{Introduction}

In males, liver cancer is the fifth most frequently diagnosed cancer worldwide and the second most frequent cause of cancerrelated mortality. In females, it is the seventh most commonly diagnosed cancer and the sixth leading cause of cancer-related mortality. Among primary liver cancers, hepatocellular carcinoma (HCC) is the major histological subtype, accounting for $70-85 \%$ of the total liver cancer burden worldwide (1). Although the prevalence of the disease remains the highest in Eastern Asia and Africa, the incidence of liver cancer has steadily increased in the Western world over the last 30-50 years (2). Over the past several years, the diagnosis and management of HCC have greatly improved. The primary curative treatment for $\mathrm{HCC}$ is surgical resection. However, many patients present with advanced stages of the disease, making surgery more difficult and less effective. This is due to the fact that the late stages of HCC are generally associated with greater invasion and metastasis, two characteristics associated with a significantly worse patient prognosis. Thus, the effective prevention of invasion and metastasis in HCC would likely be of great therapeutic value.

Increasing evidence suggests that the inhibition of cell signaling pathways can greatly influence the invasion and metastasis of HCC cells and may aid in the regulation of the disease (3-5). Previously, several independent research groups have demonstrated that Notch signaling regulates tumor cell invasion and metastasis $(6,7)$. Other studies have also indicated that Notch signaling influences the invasion of HCC cells $(8,9)$. Notch1 is a receptor that tends to be overexpressed in human HCC. Thus, Notch1 may be useful as an immunohistochemical biomarker for the detection of patients at high-risk for recurrence and with a shorter disease-specific survival (10). However, to date, the mechanisms governing the Notch1-mediated induction of the invasion of HCC cells remain poorly understood. 
In the present study, we examined the mRNA expression levels of Notch1 both in the human liver non-tumorigenic cell line, L02, and in the HCC cell lines, HepG2 and MHCC97H. Notch1 was more highly expressed in the HCC lines compared to the normal liver cell line; thus, Notch1 may play an oncogenic role in HCC. We inhibited Notch1 expression using small interfering RNA (siRNA) and assessed the effects on HCC cell line biology. Notch1 knockdown inhibited the migration and invasion of both HCC cell lines. Notch1 knockdown was also associated with the increased expression of phosphatase and tensin homolog (PTEN), both the total and phosphorylated forms, and the decreased expression of both the total and phosphorylated forms of focal adhesion kinase (FAK). Our data suggest that the Notch1-PTEN-FAK pathway may provide a new means of inhibiting the metastasis of HCC cells.

\section{Materials and methods}

Cell culture and reagents. The normal liver cell line, L02, was kindly provided by No. 3 People's Hospital Affiliated with Shanghai Jiao Tong University, Shanghai, China. The MHCC97H metastatic HCC cell line was obtained from the Liver Cancer Institute of Zhong Shan Hospital Affiliated with Fudan University, Shanghai, China. The HepG2 HCC line was obtained from the Experiment Center of the Second Affiliated Hospital of Harbin Medical University, Harbin, China. All cell lines were cultured in high-glucose Dulbecco's modified Eagle's medium (DMEM) supplemented with $10 \%$ fetal bovine serum (FBS; Biowest SAS, Nuaillé, France) and incubated in $5 \% \mathrm{CO}_{2}$ at $37^{\circ} \mathrm{C}$. Primary antibodies for Notch1, PTEN, phospho-PTEN, FAK and phospho-FAK were purchased from Cell Signaling Technology Inc. (Danvers, MA, USA). All secondary antibodies were obtained from Beijing Zhongshan Golden Bridge Biotechnology Co.Ltd. (Beijing, China). Notch1 small interfering RNA (Notch1-siRNA), control siRNA and Lipofectamine RNAiMAX were purchased from Invitrogen (Carlsbad, CA, USA). All other chemicals and solutions were purchased from Sigma-Aldrich, unless otherwise indicated.

siRNA transfection. Three putative Notch1 candidate sequences and one control sequence were designed using Oligoengine software, as previously described (11). The sequences of the siRNAs were as follows: Notch1 sequence 1 forward primer, 5'-AAC AUC AAC GAG UGG UCC AGC dTdT-3') and reverse primer, 5'-GCU GGA GCA CUC CUU GAU GUU-3'); Notch1 sequence 2 forward primer, 5'-GGG CUA ACA AAG AUA UGC ATT dTdT-3' and reverse primer, 5'-UGC AUA UCU UUG UUA GCC CTT-3'; Notch1 sequence 3 forward primer, 5'-CAG GGA GCA UGU GUA ACA UTT dTdT-3' and reverse primer, 5'-AUG UUA CAC AUG CUC CCU GTT-3'; and control sequence forward primer, 5'-CGU GCC AAC AAG UCG UAC AGA dTdT-3' and reverse primer, 5'-UGU GUA GUA CCC AGU GUU GCC-3'. All siRNA molecules were synthesized by Invitrogen (Shanghai, China). Transfection with siRNA was carried out using Lipofectamine RNAiMAX according to the manufacturer's instructions. Cells transfected with Notch1siRNA were seeded into 6-well culture plates at a density of $1 \times 10^{5}$ cells/well. Cells were allowed to grow for $24-48 \mathrm{~h}$ and were then harvested for analysis. Irrelevant control siRNA was used as a negative control (mock group) under similar conditions.
Reverse transcription polymerase chain reaction (RT-PCR) . Total RNA was extracted using TRIzol reagent (Invitrogen) according to the manufacturer's instructions and quantified by UV spectroscopy. To prepare RNA for PCR analysis, $2 \mu \mathrm{g}$ total RNA was converted into cDNA using SuperScript II reverse transcriptase (Invitrogen) with oligo(dT) (Promega, Madison, WI, USA) and random hexamer primers (Promega). PCR was performed using Taq DNA polymerase (Invitrogen). All PCR experiments were performed using the PCR system TC-XP-G (Bioer Technology Co., Ltd., Hangzhou, China). $\beta$-actin was used as an internal control for normalization. All reactions were carried out for 30 cycles. The primers used in the present study were as follows: Notch1 forward, 5'-CGA CGT CAA CGC CGT AGA T-3' and reverse, 5'-CTC CTC CCT GTT GTT CTG CATAT-3'; $\beta$-actin forward, 5'-GTC AGG TCA TCA CTA TCG GCA AT-3' and reverse, 5'-AGA GGT CTT TAC GGA TGT CAA CGT-3'. Products were analyzed by polyacrylamide gel electrophoresis.

Migration and invasion assay. A wound-healing assay was performed to assess the effects on migration. HCC cells $\left(1 \times 10^{5}\right)$ were seeded in a fibronectin (Fn)-coated 6-well plate. These cells were incubated for $24 \mathrm{~h}$. The cell monolayer was then disrupted with a pipette tip followed by 6 washes with DMEM medium to wash away any floating cells. The cells were then cultured in DMEM medium containing 2\% FBS, and images were captured at time 0 and $24 \mathrm{~h}$ after the scratch was made using an inverted microscope. Six fields for each point were recorded. For the invasion assay, Transwell assays were performed. The membranes had an $8 \mu \mathrm{m}$ diameter pore (Corning Inc., New York, NY, USA) and was coated with $200 \mu \mathrm{l}$ Matrigel at $200 \mu \mathrm{g} / \mathrm{ml}$. The membranes were incubated overnight at $4^{\circ} \mathrm{C}$. Cells $\left(2 \times 10^{4}\right)$ in $0.20 \mathrm{ml}$ serum-free DMEM were seeded in the upper chamber. The lower chamber was filled with $0.75 \mathrm{ml}$ DMEM containing 10\% FBS. After $48 \mathrm{~h}$ of incubation, the cells were removed from the upper surface of the filter by scraping with a cotton swab. Cells that had invaded and adhered to the bottom of the membrane were fixed with methanol and stained with crystal violet solution. The number of invaded cells was determined by counting the mean cell number of 5 randomly selected fields. Experiments were carried out in triplicate.

Western blot analysis. Cells were lysed in buffer containing $50 \mathrm{mmol} / \mathrm{l}$ Tris- $\mathrm{Cl}$ (pH 8.0), 0.02\% sodium azide, $1 \mathrm{mg} / 1$ aprotinin, $1 \%$ nonidet P-40, and $100 \mathrm{mg} / \mathrm{l}$ phenylmethylsulfonyl fluoride. Final protein concentrations were determined using the BCA protein assay kit (Beyotime Institute of Biotechnology, Shanghai, China) according to the manufacturer's specifications. Equal amounts of protein were separated by $10 \%$ SDS-polyacrylamide gel electrophoresis. Proteins were transferred to a nitrocellulose membrane (Amersham Biosciences, Piscataway, NJ, USA) and blocked for $2 \mathrm{~h}$ in $5 \%$ fat-free dry milk, $0.1 \%$ Tween- $20,150 \mathrm{mmol} / 1$ sodium chloride, and $50 \mathrm{mmol} / 1$ Tris. The membranes were incubated overnight at $4{ }^{\circ} \mathrm{C}$ with primary antibodies. Immunocomplexes were incubated with horseradish peroxidase-conjugated polyclonal anti-mouse or anti-rabbit $\mathrm{IgG}$ for $1 \mathrm{~h}$ at room temperature (diluted at 1:500) and visualized using an ECL kit (Amersham Biosciences) based on the manufacturer's instructions. 


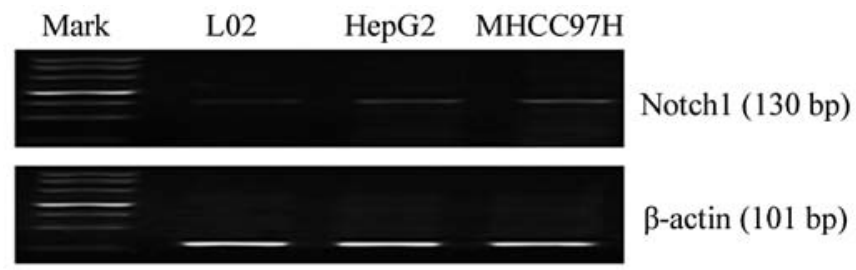

Figure 1. Notch1 gene expression in the human liver non-tumorigenic cell line, L02, and the hepatocellular carcinoma (HCC) cell lines, HepG2 and MHCC97H Notch1 was expressed in all 3 cell lines, but its expression was increased in the 2 HCC lines compared to L02. $\beta$-actin was used as a normalization control.
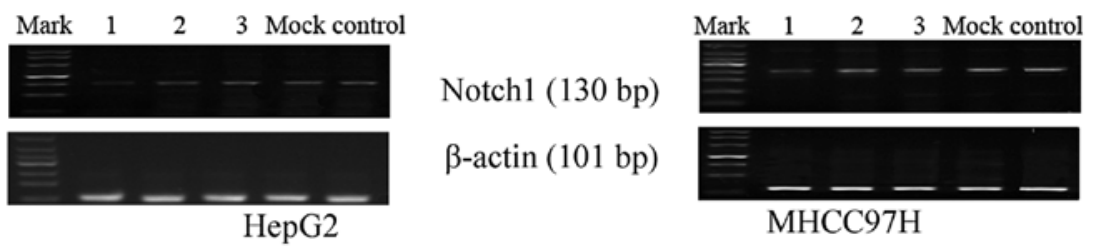

Figure 2. Knockdown efficiency of 3 different Notch1 siRNA sequences was tested in both HepG2 and MHCC97H cell lines by RT-PCR. Comparisons were made to cells that were either mock-transfected or transfected with a non-specific control siRNA. Notch1 siRNA 1 showed the best degree of knockdown and was used in subsequent experiments. $\beta$-actin was used as a normalization control.

A

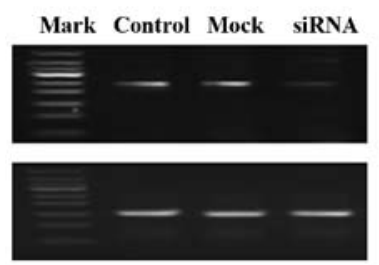

HepG2

B

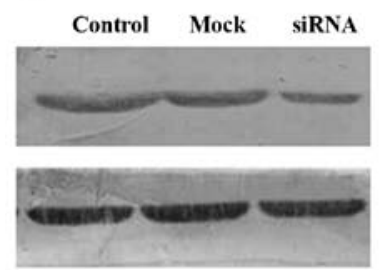

HepG2
Mark Control Mock siRNA

Notchl (130 bp)

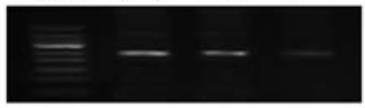

$\beta$-actin (101 bp)

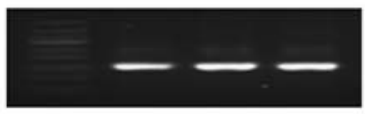

MHCC97H

Notchl (120 kDa)

$\beta$-actin (42 kDa)

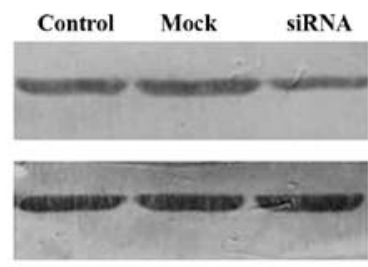

MHCC97H

Figure 3. Knockdown of Notch1 by siRNA analyzed by RT-PCR and western blot analysis in the hepatocellular carcinoma (HCC) cell lines, HepG2 and MHCC97H. (A) Notch1 mRNA expression in HepG2 and MHCC97H cells transfected with siRNA; Notch1 expression was decreased compared to the control and mock groups. (B) Notch1 was also decreased at the protein level following transfection with siRNA in both cell lines.

Statistical analysis. Each experiment was repeated at least 3 times. The data are presented as the means \pm standard deviation (SD). The results were analyzed by one-way analysis of variance. All statistical analyses were performed using SPSS 13.0 software (SPSS Inc., Chicago, IL, USA). A value of P<0.05 was considered to indicate a statistically significant difference.

\section{Results}

Notchl expression is elevated in HCC cells. We first examined the baseline mRNA expression level of Notch1 in the L02, HepG2 and MHCC97H cell lines by RT-PCR. The Notch1 transcript was highly expressed in the HCC cells compared to the normal liver cell line (Fig. 1). Based on the gene expression data, we hypothesized that Notch1 expression may be associated with the invasion of HCC cells.
Notch1 silencing using siRNA. The HepG2 and MHCC97H cells, which have relatively high expression levels of Notch1, were transiently transfected with Notch1-siRNA or mock siRNA. We designed 3 candidate Notch1-specific sequences and one control sequence (mock). RT-PCR was performed to assess the knockdown efficiency of the candidate siRNAs. As illustrated in Fig. 2, the candidate sequence 1 most effectively inhibited Notch1 mRNA expression compared to the control. Thus, this siRNA was selected for use in the subsequent experiments. Notch1 mRNA and protein expression was quantified and analyzed by RT-PCR and western blot analysis, respectively, $72 \mathrm{~h}$ following transfection with siRNA. Compared to the control (no siRNA) and mock-transfected cells (negative control siRNA), Notch1 mRNA and protein expression was markedly decreased in the cells transfected with Notch1-siRNA (Fig. 3). 
A

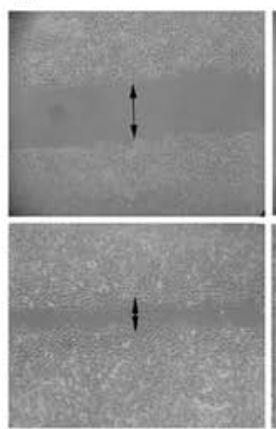

Control

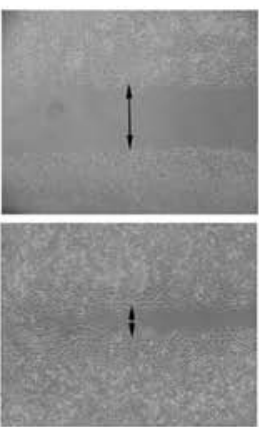

Mock

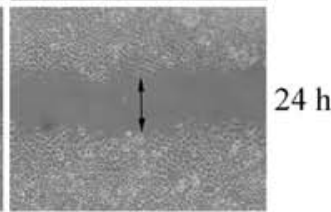

siRNA
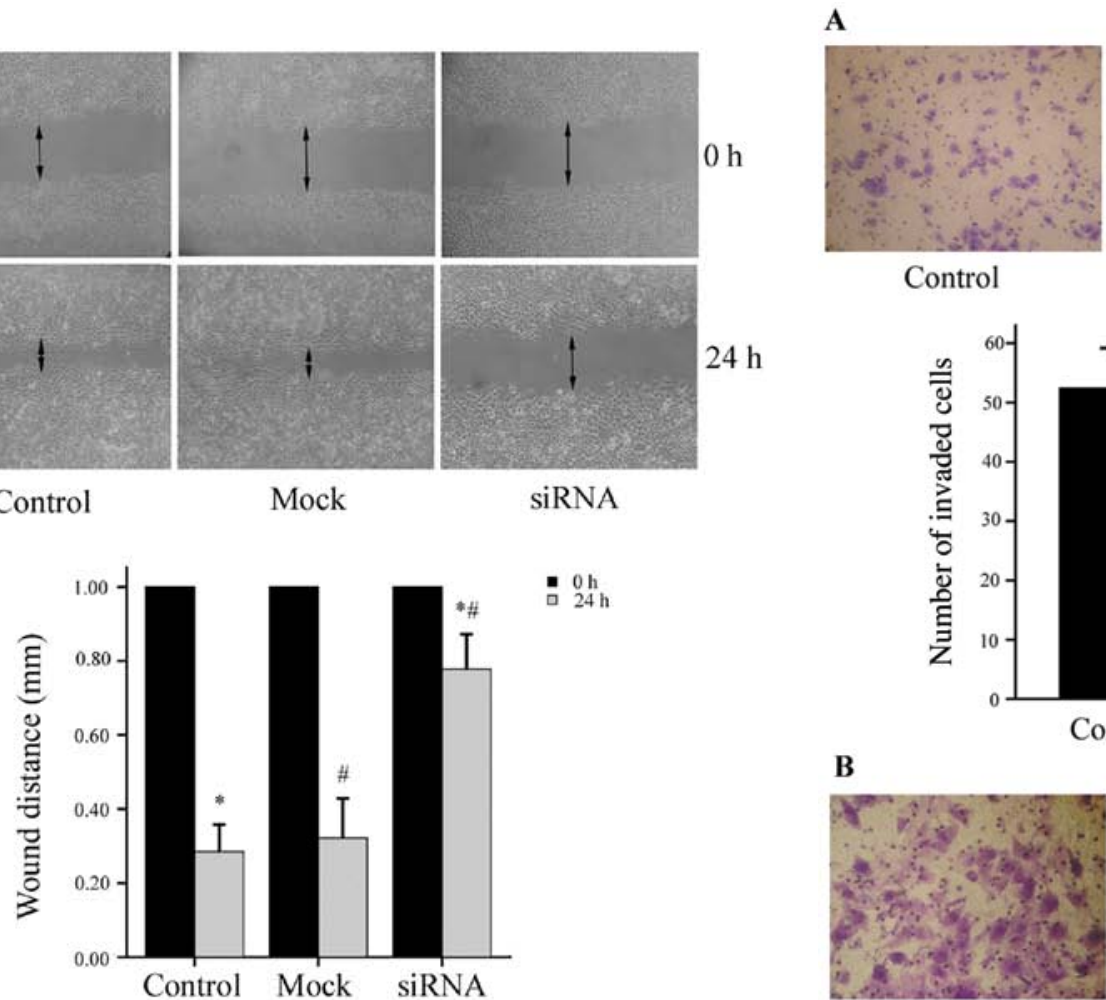

- $24 \mathrm{~h}$

B

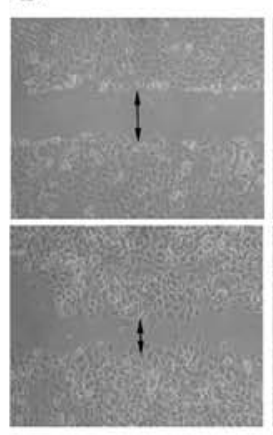

Control

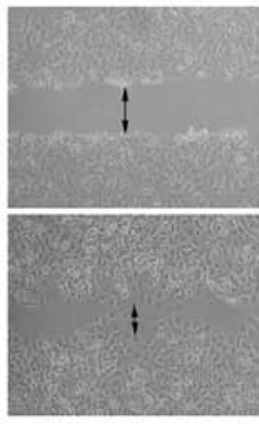

Mock

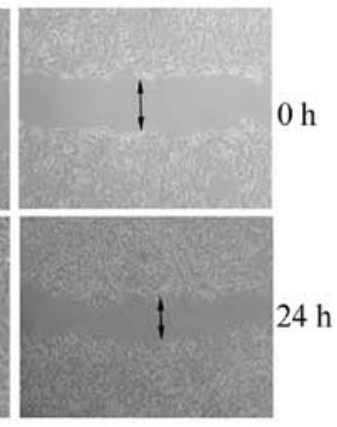

SiRNA

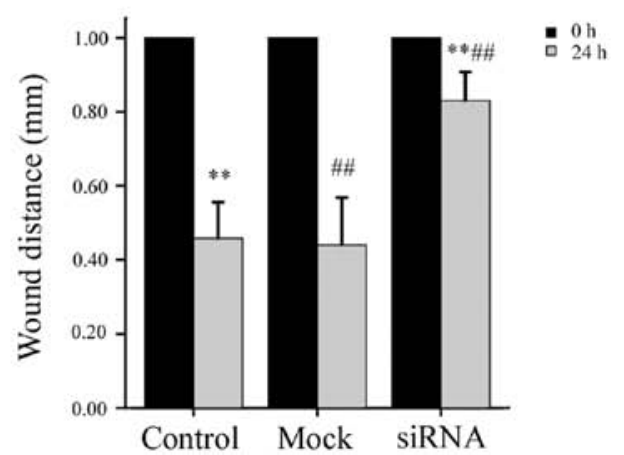

Figure 4. Downregulation of Notch1 by siRNA inhibits cell migration in the hepatocellular carcinoma (HCC) cell lines, HepG2 and MHCC97H. (A) Representative images from the wound-healing assay performed on HepG2 cells showing that the migration of the siRNA-transfected group was inhibited compared to the control and mock groups. Wound size was as follows: siRNA group, $0.78 \pm 0.09 \mathrm{~mm}$; mock group, $0.32 \pm 0.11 \mathrm{~mm}$; control group, $0.29 \pm 0.07 \mathrm{~mm} ;{ }^{*} \mathrm{P}<0.01$ between the indicated groups; control vs. siRNA, "P<0.01 between the indicated groups; mock vs. siRNA. All data are expressed as the means $\pm \mathrm{SD}$; $(\mathrm{n}=6)$ for each group. (B) Wound-healing assay of MHCC97H cells was consistent with the results from HepG2 cells. Wound size was as follows: siRNA group, $0.83 \pm 0.07 \mathrm{~mm}$; mock group, $0.44 \pm 0.13 \mathrm{~mm}$; control group, $0.46 \pm 0.10 \mathrm{~mm} ;{ }^{* *} \mathrm{P}<0.01$ between the indicated groups; control vs. siRNA, ${ }^{\# \#} \mathrm{P}<0.01$ between the indicated groups; mock vs. siRNA. All data are expressed as the means $\pm \mathrm{SD} ;(\mathrm{n}=6)$ for each group.
Control

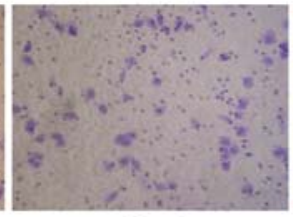

Mock

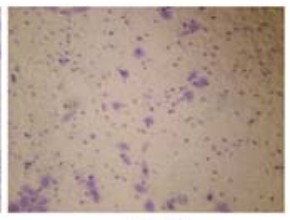

siRNA

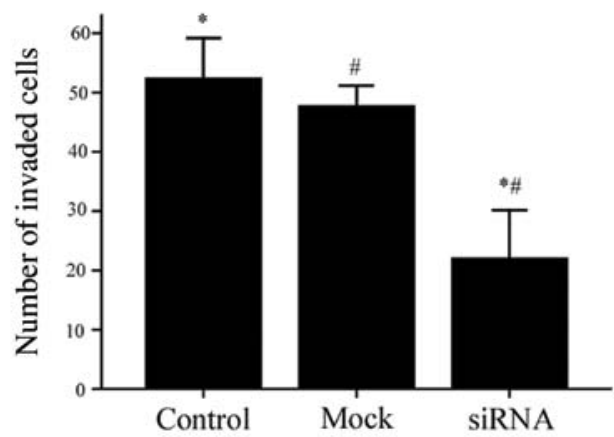

B

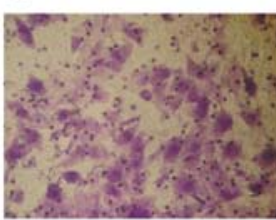

Control

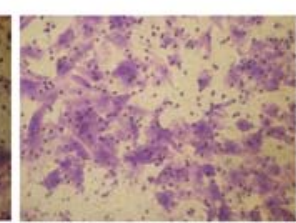

Mock

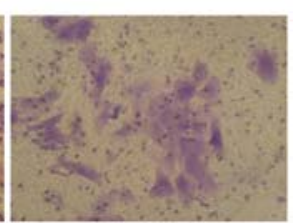

siRNA

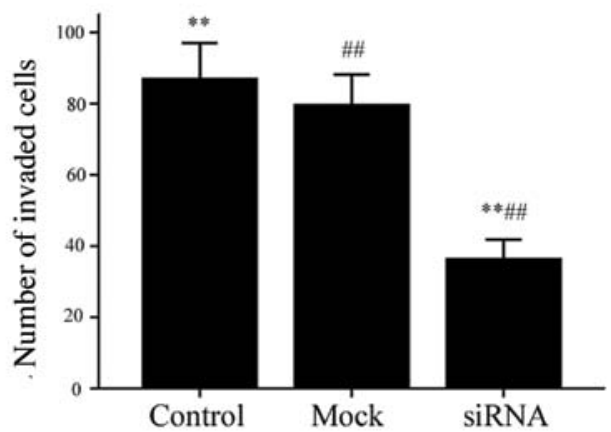

Figure 5. siRNA-mediated downregulation of Notch1 inhibits the invasion of hepatocellular carcinoma (HCC) cells. (A) Representative images showing that HepG2 cell migration was decreased in the Notch1 siRNA group compared to the control and mock groups. The number of cells that invaded through the membrane was as follows: siRNA group, $22 \pm 8.19$; control group, $52.33 \pm 6.81$; mock group, $47.67 \pm 3.51$; all data are expressed as the means $\pm S D$; $(n=3)$ for each group; "P<0.01 between the indicated groups; control vs. siRNA, ${ }^{\sharp} \mathrm{P}<0.01$ between the indicated groups; mock vs. siRNA. (B) Results for MHCC97H cells were consistent with the data for the HepG2 cells. Representative images are shown. All data are expressed as the means $\pm \mathrm{SD}$; $(\mathrm{n}=3)$ for each group; ${ }^{* *} \mathrm{P}<0.01$ between the indicated groups; control vs. siRNA, ${ }^{\# \#} \mathrm{P}<0.01$ between the indicated groups; mock vs. siRNA.

Downregulation of Notch1 expression suppresses HCC cell migration and invasion. To determine whether the down-regulation of Notch1 expression affects the migratory ability of the HepG2 and MHCC97H cells, we performed a wound-healing assay. The migration of HepG2 cells was significantly inhibited by Notch1 knockdown. The size of the wound in the Notch1siRNA group was $0.78 \pm 0.09 \mathrm{~mm}$, which was significantly larger than the size of the wound in either the mock-transfected group $(0.32 \pm 0.11 \mathrm{~mm})$ or the control group $(0.29 \pm 0.07 \mathrm{~mm})$ $(\mathrm{P}<0.01, \mathrm{n}=6)$. Similar results were obtained for the MHCC97H cells; the size of the wound in the Notch1-siRNA group was 


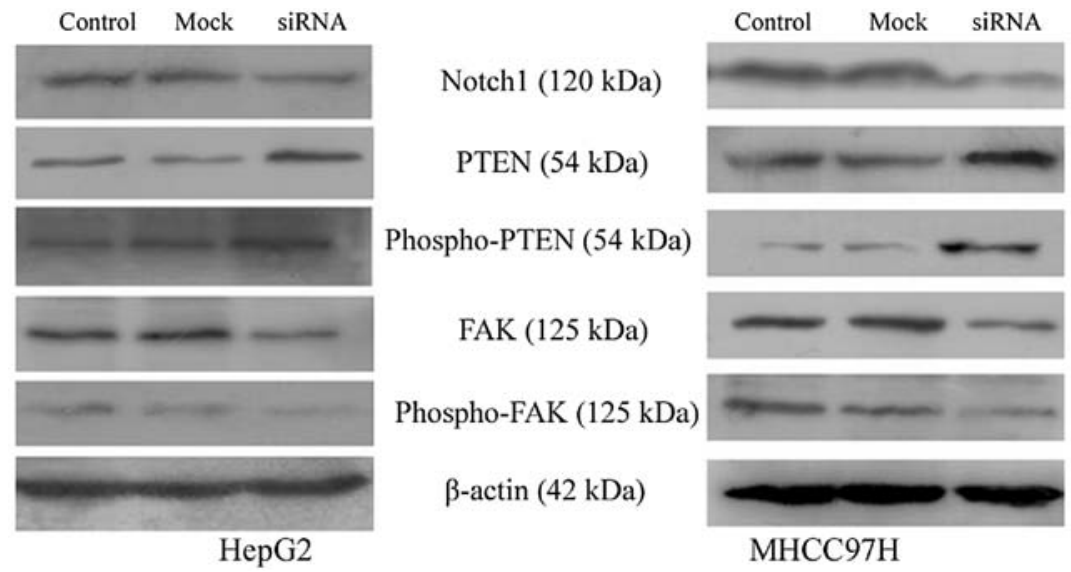

Figure 6. Levels of Notch1 in HepG2 and MHCC97H cells were downregulated following transfection with siRNA. This was followed by western blot analysis of phosphatase and tensin homolog (PTEN), phospho-PTEN, focal adhesion kinase (FAK) and phospho-FAK-proteins known to be involved in the invasion of hepatocellular carcinoma (HCC) cells. Following knockdown, the levels of PTEN and phospho-PTEN were increased and those of FAK and phospho-FAK were decreased in both cell lines.

$0.83 \pm 0.07 \mathrm{~mm}$, compared to $0.44 \pm 0.13 \mathrm{~mm}$ in the mock group and $0.46 \pm 0.10 \mathrm{~mm}$ in the control group $(\mathrm{P}<0.01, \mathrm{n}=6)$ (Fig. 4). These results demonstrate that the siRNA-mediated knockdown of Notch1 inhibits the migration of HepG2 and $\mathrm{MHCC} 97 \mathrm{H}$ cells. The results from Transwell Matrigel invasion assays were consistent with our wound-healing assay results. As shown in Fig. 5, the number of HepG2 cells that successfully invaded through the chamber was lower in the Notch1-siRNA group $(22 \pm 8.19)$ compared to both the mock group $(47.67 \pm 3.51)$ and the control group $(52.33 \pm 6.81)(\mathrm{P}<0.01)$. The same was true for the MHCC97H cells (Notch1-siRNA, 36.33 \pm 5.51 ; mock, 79.67 \pm 8.51 ; control, 87.00 \pm 10.15 ; $\mathrm{P}<0.01$ ). Taken together, our data support a role for Notch1 in the migratory and invasive capabilities of HepG2 and MHCC97H cells.

Downregulation of Notchl alters the expression of PTEN and FAK. PTEN is a critical tumor suppressor gene located on human chromosome 10q23 (12). FAK (13) has been shown to be an important mediator of cell adhesion, growth, proliferation, survival, angiogenesis and migration, all of which are often disrupted in cancer cells. PTEN interacts with FAK and reduces its tyrosine phosphorylation (14). As shown in Fig. 6, the downregulation of Notch1 in HepG2 and MHCC97H cells increased the expression of both PTEN and phospho-PTEN and decreased the expression of FAK and phospho-FAK compared to the control and mock-transfected cells.

\section{Discussion}

Invasion and metastasis are the primary cause of mortality from HCC. Thus, novel therapies that specifically inhibit these processes are critical. The inhibition of cell signaling pathways for antitumor efficacy has shown great promise (15). It has recently been demonstrated that the persistent activation of Notch signaling is associated with liver malignancies (10). In humans, the Notch family of transmembrane proteins consists of four receptors (Notch1 through Notch4). Importantly, the high expression of Notch1 in HCC has been shown to correlate with an advanced TNM stage and blood vessel infiltration (8). In this study, we found that Notch1 expression was elevated in two HCC cell lines compared to normal liver cells. Thus, Notch1 may be a potential therapeutic target in HCC.

To elucidate the functional relevance of Notch1 in HCC, we modulated Notch1 expression levels in HCC cell lines using siRNA. Clinically, Notch signaling can be inhibited by one of three ways. First, the activation of the Notch receptor can be inhibited by the use of gamma-secretase inhibitors (GSIs). Second, ligand binding to the Notch receptor can be blocked by monoclonal antibodies. Finally, the transcriptional activity of the Notch intracellular domain can be inhibited using blocking peptides. Inhibition by siRNA, as used in the present study, is likely most similar to inhibition via the prevention of ligand binding. The use of siRNA tends to show greater specificity than GSIs, which are not cell-type specific. Moreover, GSIs have a considerable toxicity profile. Our results demonstrated that the downregulation of Notch1 expression in HepG2 and MHCC 97H cells by siRNA suppressed HCC cell migration and invasion. Recent data provided by others supports our findings. For example, Zhou et al (9) demonstrated that GSIs suppress the invasion of HCC cells; however, Notch1 was not analyzed in their study. Our data support a new role for Notch1 in HCC cell invasion.

Notably, we found that the total and phosphorylated levels of PTEN were increased in the HCC cells following Notch1 depletion. Consistent with this finding, GSI treatment has been shown to upregulate PTEN protein expression in the primarylike leukemia cell line, TAIL7 (16). Palomero et al (17) also reported that Notch1 negatively regulates PTEN at the transcriptional level. PTEN protein was originally identified as a potent tumor suppressor (18-21). PTEN reduces the rates of migration through several mechanisms. One mechanism involves effects on cell adhesion. FAK is a key molecule implicated in integrin and growth factor-mediated signaling, and plays an important role in cell adhesion. FAK has also been shown to interact with PTEN to influence tumor cell invasion (14). FAK is an important tyrosine kinase that regulates tumor invasion and survival (22-24), and it is significantly overexpressed in HCC (25-27). Growing evidence indicates that the inhibition of FAK may be a useful therapy against cancer cell metastasis (28-30). PTEN is a phosphatase that can negatively 
regulate FAK tyrosine phosphorylation $(31,32)$. The decreased phosphorylation of FAK mediated by PTEN inhibits cellular migration, spreading and adhesion. In the present study, we demonstrated that the downregulation of Notch1 by siRNA in HepG2 and MHCC97H cells increased PTEN expression and decreased the expression of FAK and phospho-FAK. We hypothesized that the downregulation of Notch1 may inhibit HCC through the upregulation of PTEN and the subsequent inactivation of FAK. In conclusion, Notch1-siRNA affects the balance of phospho-FAK and FAK by increasing the levels of PTEN and phospho-PTEN; in effect, these molecular changes help suppress HCC invasion. Whether or not FAK phosphorylation is inversely correlated with PTEN levels in HCC cell lines transfected with Notch1-siRNA requires additional research. We suggest that the Notch1-PTEN-FAK signaling axis may be a critical determinant of liver cancer metastasis.

In conclusion, our results demonstrate that the downregulation of Notch1 by siRNA in HepG2 and MHCC $97 \mathrm{H}$ cells decreases cell invasion. Furthermore, decreasing Notch1 expression upregulates PTEN and phospho-PTEN and downregulates FAK and phospho-FAK expression. The Notch1-PTEN-FAK signaling axis may be critical for HCC invasion and may represent a novel therapeutic target in the disease to inhibit metastasis.

\section{Acknowledgements}

We thank the No. 3 People's Hospital Affiliated with Shanghai Jiao Tong University and the Liver Cancer Institute of Zhong Shan Hospital Affiliated with Fudan University for kindly providing the human liver non-tumor cell line (L02) and the HCC cell line (MHCC97H). The present study was supported by grants from the Postdoctoral Science Foundation of China (no. 2011M500686) and the Science and Technology Research Foundation of Heilongjiang Province Department of Education of China (no. 12521178).

\section{References}

1. Siegel R, Ward E, Brawley O and Jemal A: Cancer statistics, 2011: the impact of eliminating socioeconomic and racial disparities on premature cancer deaths. CA Cancer J Clin 61: 212-236, 2011

2. Marquardt JU, Galle PR and Teufel A: Molecular diagnosis and therapy of hepatocellular carcinoma (HCC): an emerging field for advanced technologies. J Hepatol 56: 267-275, 2012.

3. Wang YH, Dong YY, Wang WM, et al: Vascular endothelial cells facilitated $\mathrm{HCC}$ invasion and metastasis through the Akt and NF- $\kappa$ B pathways induced by paracrine cytokines. J Exp Clin Cancer Res 32: 51, 2013.

4. Chen JS, Huang XH, Wang Q, et al: Sonic hedgehog signaling pathway induces cell migration and invasion through focal adhesion kinase/AKT signaling-mediated activation of matrix metalloproteinase (MMP)-2 and MMP-9 in liver cancer. Carcinogenesis 34: 10-19, 2013.

5. Liu L, Dai Y, Chen J, et al: Maelstrom promotes hepatocellular carcinoma metastasis by inducing epithelial-mesenchymal transition by way of $\mathrm{Akt} / \mathrm{GSK}-3 \beta /$ Snail signaling. Hepatology 59 : 531-543, 2014.

6. Bolós V, Grego-Bessa J and de la Pompa J L: Notch signaling in development and cancer. Endocr Rev 28: 339-363, 2007.

7. Hu YY, Zheng MH, Zhang R, Liang YM and Han H: Notch signaling pathway and cancer metastasis. Adv Exp Med Biol 727: 186-198, 2012.

8. Wang X Q, Zhang W, Lui E L, et al: Notch1-Snail1-E-cadherin pathway in metastatic hepatocellular carcinoma. Int J Cancer 131: E163-E172, 2012.
9. Zhou L, Wang DS, Li QJ, Sun W, Zhang Y and Dou KF: Downregulation of the Notch signaling pathway inhibits hepatocellular carcinoma cell invasion by inactivation of matrix metalloproteinase- 2 and -9 and vascular endothelial growth factor. Oncol Rep 28: 874-882, 2012.

10. Ahn S, Hyeon J and Park C K: Notchl and Notch4 are markers for poor prognosis of hepatocellular carcinoma. Hepatobiliary Pancreat Dis Int 12: 286-294, 2013.

11. Fox V, Gokhale PJ, Walsh JR, Matin M, Jones M and Andrews PW: Cell-cell signaling through NOTCH regulates human embryonic stem cell proliferation. Stem Cells 26: 715-723, 2008.

12. Singh $G$ and Chan AM: Post-translational modifications of PTEN and their potential therapeutic implications. Curr Cancer Drug Targets 11: 536-547, 2011.

13. Golubovskaya VM: Focal adhesion kinase as a cancer therapy target. Anticancer Agents Med Chem 10: 735-741, 2010.

14. Zhang L, Yu Q, He J and Zha X: Study of the PTEN gene expression and FAK phosphorylation in human hepatocarcinoma tissues and cell lines. Mol Cell Biochem 262: 25-33, 2004.

15. Wong R, Frenette C and Gish R: Hepatocellular carcinoma: locoregional and targeted therapies. Gastroenterol Clin North Am 40: 599-610, 2011.

16. Silva A, Jotta P Y, Silveira A B, et al: Regulation of PTEN by CK2 and Notch1 in primary T-cell acute lymphoblastic leukemia: rationale for combined use of CK2-and $\gamma$-secretase inhibitors. Haematologica 95: 674-678, 2010.

17. Palomero T, Sulis ML, Cortina M, et al: Mutational loss of PTEN induces resistance to NOTCH1 inhibition in T-cell leukemia. Nat Med 13: 1203-1210, 2007.

18. Wang L, Wang WL, Zhang Y, Guo SP, Zhang J and Li QL: Epigenetic and genetic alterations of PTEN in hepatocellular carcinoma. Hepatol Res 37: 389-396, 2007.

19. Chow LM and Baker SJ: PTEN function in normal and neoplastic growth. Cancer Lett 241: 184-196, 2006.

20. Hu TH, Huang CC, Lin PR, et al: Expression and prognostic role of tumor suppressor gene PTEN/MMAC1/TEP1 in hepatocellular carcinoma. Cancer 97: 1929-1940, 2003.

21. Dong-Dong L, Xi-Ran Z and Xiang-Rong C: Expression and significance of new tumor suppressor gene PTEN in primary liver cancer. J Cell Mol Med 7: 67-71, 2003.

22. Thanapprapasr D, Hu W, Sood AK and Coleman RL: Moving beyond VEGF for anti-angiogenesis strategies in $\mathrm{g}$ ynecologic cancer. Curr Pharm Des 18: 2713-2719, 2012.

23. Bai X, Wang J, Zhang L, et al: Prostaglandin $\mathrm{E}_{2}$ receptor EP1-mediated phosphorylation of focal adhesion kinase enhances cell adhesion and migration in hepatocellular carcinoma cells. Int J Oncol 42: 1833-1841, 2013.

24. Zhang $\mathrm{C}$, He H, Zhang H, et al: The blockage of Ras/ERK pathway augments the sensitivity of SphK1 inhibitor SKI II in human hepatoma HepG2 cells. Biochem Biophys Res Commun 434: 35-41, 2013.

25. Chen JS, Huang XH, Wang Q, et al: FAK is involved in invasion and metastasis of hepatocellular carcinoma. Clin Exp Metastasis 27: 71-82, 2010.

26. Lau GM, Lau GM, Yu GL, et al: Expression of Src and FAK in hepatocellular carcinoma and the effect of Src inhibitors on hepatocellular carcinoma in vitro. Dig Dis Sci 54: 1465-1474, 2009.

27. Han S, Han L, Yao Y, Sun H, Zan X and Liu Q: Activated hepatic stellate cells promote hepatocellular carcinoma cell migration and invasion via the activation of FAK-MMP9 signaling. Oncol Rep 31: 641-648, 2014.

28. Ko BS, Jan YJ, Chang TC, et al: Upregulation of focal adhesion kinase by $14-3-3 \varepsilon$ via NFKB activation in hepatocellular carcinoma. Anticancer Agents Med Chem 13: 555-562, 2013.

29. Sheng SL, Liu JJ, Dai YH, Sun XG, Xiong XP and Huang G: Knockdown of lactate dehydrogenase A suppresses tumor growth and metastasis of human hepatocellular carcinoma. FEBS J 279: 3898-3910, 2012.

30. Xu HY, Qian AR, Shang P, et al: siRNA targeted against HAb18G/ CD147 inhibits MMP-2 secretion, actin and FAK expression in hepatocellular carcinoma cell line via ERK1/2 pathway. Cancer Lett 247: 336-344, 2007.

31. Chetram MA and Hinton CV: PTEN regulation of ERK1/2 signaling in cancer. J Recept Signal Transduct Res 32: 190-195, 2012.

32. Tamura M, Gu J, Danen E H J, et al: PTEN interactions with focal adhesion kinase and suppression of the extracellular matrix-dependent phosphatidylinositol3-kinase/Akt cell survival pathway. J Biol Chem 274: 20693-20703, 1999. 